\title{
An Efficient Combination of Dispatch Rules for Job-shop Scheduling Problem
}

\author{
Tatsunobu Kawai, Yasutaka Fujimoto \\ Department of Electrical and Computer Engineering, Yokohama National University, Yokohama 240-8501 JAPAN \\ kawai@fujilab.dnj.ynu.ac.jp, fujimoto@ynu.ac.jp \\ http://www.fujilab.dnj.ynu.ac.jp
}

\begin{abstract}
This paper present new combinations of dispatch scheduling for Job Shop Scheduling Problem. The Job Shop Scheduling Problem is one of the NP hard optimization problems, and it is difficult to obtain the exact optimal solution. Scheduling methods based on the dispatch rule are a set of efficient approximate methods. In this paper, by combining several dispatch rules, we have proposed three new rules. The first rule is the rule that combines two simple dispatch rules which are often adopted in actual production systems. The proposed rule gives better result than that of any single dispatch rule. The second rule is the rule that keeps balance of remaining load of all machines. In this rule, the weight is decided in early stage of the schedule. It gives good solutions with high probability. The third rule is the rule that predetermines the mixing ratio, which accompanies a sequence of operations. This rule often gives better results than any other dispatch rules.
\end{abstract}

\section{INTRODUCTION}

In recent years, multi-item small-lot-sized production becomes popular since it is possible to meet wide variety of consumer needs and shortened life cycle of goods. A scheduling problem for this type of production system becomes very complex.

Many real scheduling problems are represented as Job Shop Scheduling Problem (JSSP). Because almost all scheduling problems, comprising JSSP, are the NP hard optimization problem, an efficient approximate method is required. The approximate methods are categorized in heuristics and meta-heuristics. Meta-heuristics are methods that search many solutions simultaneously by simple calculation and choose the best one in the solutions. This category includes Genetic-Algorithm and SimulatedAnnealing.

Heuristics depend on experience and expertise like dispatch rules. Since the dispatch rules are based on experience and expertise, some of the dispatch rules are not effective for some problems, i. e., conventionally, there is no dispatch rule that always gives good solution for any problem. Therefore, it is useful to develop a new rule that can be applied to many problems. A survey of dispatch rules is found in [1].

In this paper, by combining several dispatch rules, we propose a new rule that can give shorter makespan than that of other general dispatch rules for many problems.

\section{Conventional Dispatch Rules}

\section{A. Conventional Dispatch Rules}

The below list shows various type of conventional dispatch rules. These rules are widely adopted at many factories .

- SPT (Shortest Processing Time)

- select a job which has the shortest processing time

- LPT (Longest Processing Time)

- select a job which has the longest processing time

- MWKR (Most WorK Remaining)

- select a job which has longest total processing time remaining

- LOPN (Least OPeration Numbers)

- select a job which has the least operation number

- SLACK

- select a job which has the shortest due date

Our new scheduling method is based on these dispatch rules.

\section{B. Dependence of Rules on Properties of Problems}

An effectiveness of a dispatch rule tends to depend on characteristics of structure of problems. Although this fact has not been theoretically discussed well in past works, it is generally understood to be possible. In order to develop a new combination of the rules, it is effective to investigate how each dispatch rule depends on properties of the problems.

In numerical simulations, we adopt four dispatch rules for thirteen bench mark problems which are given by the OR-Library[2]. The OR-Library is a collection of test data sets for a variety of Operations Research (OR) problems these bench mark problems are regarded as representatives of the JSSP.

The simulation results are shown in Table I. In the Table I, a mark "**" denotes the shortest makespan among the four rules. From the Table I, MWKR gives many good and stable makespans than the other three rules. SPT and LOPN give some good makespans. On the other hand, LPT gives worse makespans. The Table I shows that the effective rule differs depending on properties of the problems.

\section{Sensitivity of Rules}

When two or more dispatch rules are combined and different priorities are mixed together, a top prior job under 


\begin{tabular}{|c|c|c|c|c|}
\hline & \multicolumn{4}{|c|}{ Rule } \\
\hline problem & SPT & LPT & MWKR & LOPN \\
\hline abz5 & ${ }^{* *} 1352$ & 1586 & 1369 & 1356 \\
\hline abz6 & 1097 & 1207 & ${ }^{* *} 987$ & 989 \\
\hline ft10 & ${ }^{* *} 1074$ & 1295 & 1108 & 1179 \\
\hline la16 & 1156 & 1229 & ${ }^{* *} 1054$ & 1173 \\
\hline la17 & 924 & 1082 & ${ }^{* *} 846$ & 928 \\
\hline la18 & 981 & 1114 & ${ }^{* *} 970$ & 972 \\
\hline la19 & ${ }^{* *} 940$ & 1062 & 1013 & 975 \\
\hline la20 & 1000 & 1272 & ${ }^{* *} 964$ & 1160 \\
\hline orb01 & 1394 & 1410 & 1354 & ${ }^{* *} 1326$ \\
\hline orb02 & 1175 & 1293 & ${ }^{* *} 1047$ & 1157 \\
\hline orb03 & $* * 1179$ & 1430 & 1247 & 1404 \\
\hline orb04 & 1236 & 1427 & 1172 & ${ }^{* *} 1132$ \\
\hline orb05 & 1128 & 1099 & 1173 & $* * 1055$ \\
\hline
\end{tabular}

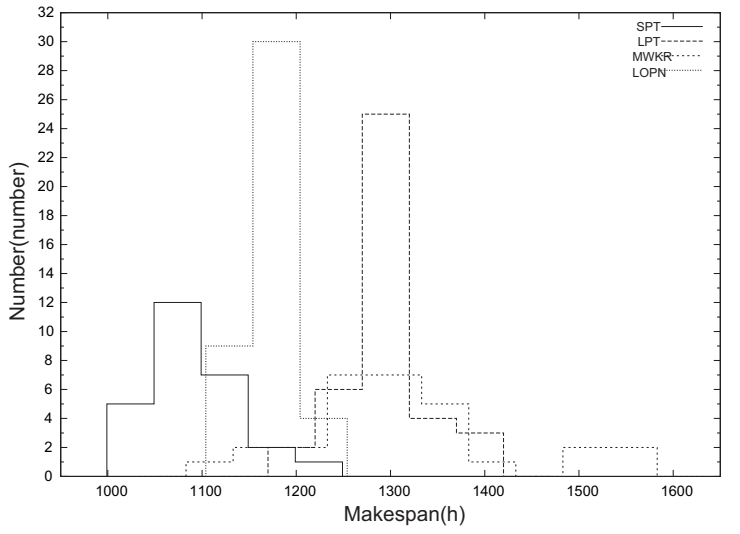

Fig. 1. Distribution of makespan when a second priority job is selected only once (ft10)

a method to determine weight of each rule in early stage during the scheduling.

the mixed dispach rule may differ from top prior jobs under the original dispach rules. Namely, from the viewpoints of the original priorities, a less prior job can be selected. In this section, we investigate the makespan for the case that a machine selects a job of second priority only once under a single dispach rule. This analysis shows a sensitivity of a single dispach rule. If the makespan varies much, we can say that rule is sensitive to the priority. In this case, the selection of a less prior job may yield improvement of the makespan.

Various scenario are tested for the problem ft10 using four dispach rules SPT, LPT, MWKR, and LOPN. There are many conflicting points during scheduling, that is, a second prior job is selected once at a randomly selected stage of scheduling. The histogram in Fig. 1 shows the number of realizations falling into each ranges of makespans. Results using usual priority are also shown in Table I. From Fig. 1, the variances of the makespan of SPT and LOPN are small. On the other hand, and MWKR has the large variance. As the result, it is possible to improve the makespan by introducing an other priority attribute in addition to the basic rule.

\section{Combination of Rules}

\section{A. Proposed Method I}

Since a processing sequence of the conventional dispatch schedule is decided by the simple priority rule, there is no general rule for every scheduling problem. Therefore, it is useful to develop a combined rule that gives more flexible and better results to many problems.

In this paper, we propose a novel rule based on a combination of some dispatch rules. From Fig. 1, we can improve the schedule and the makespan by selecting a job of lower priority in some cases. Combination of some dispatch rules changes criterion of the priority. Therefore, using a combined rule can shorten the makespan, and the combined rule can be applied to many scheduling problems when a proper weight of each rules is selected. We propose

\section{A.1 SPT and SLACK}

From the results of previous section, SPT and MWKR are selected as candidates for the combination of rules. Actually we adopt SPT and SLACK. SLACK can give the same result as MWKR when all jobs have a same due date. The proposing rule is defined as

$$
\begin{aligned}
\min _{j} S_{k j}=h_{1} p_{k j}+ & \left(1-h_{1}\right) \frac{s_{j}}{J_{k}}, \\
0 & <h_{1}<1,
\end{aligned}
$$

where $p_{k j}$ denotes processing time of job $j$ in machine $k$ and $s_{j}$ is SLACK of job $j$ defined as

$$
s_{j}=d_{j}-l_{j}-t,
$$

where $d_{j}$ denotes a due date of job $j, l_{j}$ denotes a remaining processing time of job $j$, and $t$ is a present time, respectively. $J_{k}$ denotes a number of remaining jobs of machine $k$. For machine $k$, this combination of rules of SPT and SLACK selects a job that has the smallest $S_{k j}$. We call this rule method $I$.

\section{A.2 Simulation of Method I}

To confirm the effectiveness of the method I, we examined the makespan when parameter $h_{1}$ changes from 0 to 1.

Thirteen bench mark problems[2] are adopted for the simulations. Due dates are set for all of these problems. The simulation results are shown in Fig. 2 and Table II. In Table II, conventional rule means the shortest makespan among the four rules (SPT, LPT, MWKR and LOPN). In Fig. 2, 5/10, 7/10, 9/10 mean the corresponding lap time of processing. For example, $5 / 10$ is the time when a half of total process is finished.

From Table II, the combined rule gives the best result in all problems, when a proper parameter $h_{1}$ is given.

From Fig. 2, the proper parameter $h_{1}$ changes depending on the stage of schedule. For example, $h_{1} \approx 0.6$ gives the 


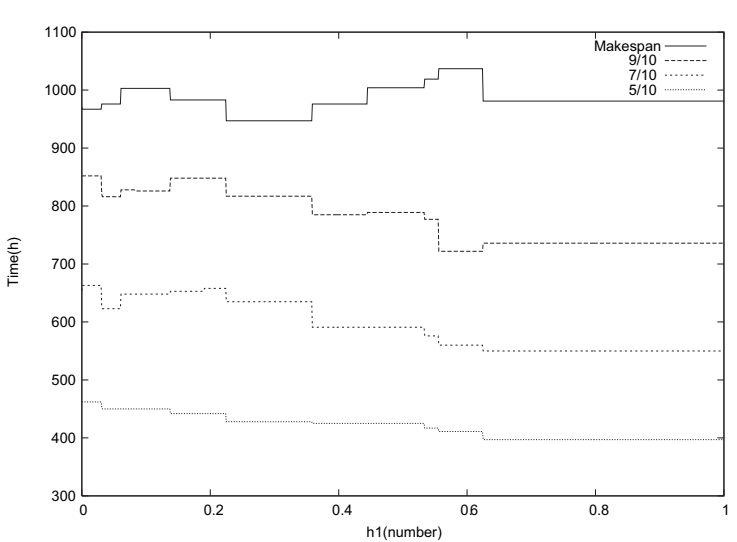

Fig. 2. Parameter $h_{1}$ v.s. processing time (la18)

TABLE II

MAKESPAN OF METHOD I

\begin{tabular}{|l|c|cl|}
\hline problem & method I & \multicolumn{2}{|c|}{ conventional rule } \\
\hline abz5 & 1281 & 1352 & (SPT) \\
\hline abz6 & 980 & 987 & (MWKR) \\
\hline ft10 & 1007 & 1074 & (SPT) \\
\hline la16 & 998 & 1054 & (MWKR) \\
\hline la17 & 827 & 846 & (MWKR) \\
\hline la18 & 947 & 970 & (MWKR) \\
\hline la19 & 928 & 940 & (SPT) \\
\hline la20 & 949 & 964 & $(\mathrm{MWKR})$ \\
\hline orb01 & 1247 & 1326 & $(\mathrm{LOPN})$ \\
\hline orb02 & 977 & 1047 & $(\mathrm{MWKR})$ \\
\hline orb03 & 1152 & 1179 & $(\mathrm{SPT})$ \\
\hline orb04 & 1084 & 1132 & $(\mathrm{LOPN})$ \\
\hline orb05 & 1031 & 1055 & $(\mathrm{LOPN})$ \\
\hline
\end{tabular}

best result when 9/10 of the schedule have been completed. However, it finally gives the worst makespan. The reason is that the remaining jobs collect to a certain machine. In order to decide the parameter $h_{1}$ in early stage of schedule, it is expected that we need to keep a balance of remaining operation time of all machines.

\section{B. Proposed Method II}

To keep remaining loads in all machines balanced, a new factor is introduced.

\section{B.1 Uniform Job Remaining}

To keep a balance of remaining operation time of all machines, the following index $U_{j}$ is introduced. A job with least $U_{j}$ should be selected for machine $k . U_{j}$ is defined as

$$
U_{k j}=\left|\left(l_{k}-p_{k j}\right)-\frac{l_{1}+l_{2}+\cdots+\left(l_{k}-p_{k j}\right)+\cdots+l_{m}}{m}\right|,
$$

where $l_{k}$ denotes a remaining operation time of a machine $k$ and $p_{k j}$ denotes a processing time of job $j$ in machine $k$, respectively. This attribute is named UJR (Uniform Job Remaining) in this paper. The combination of rules of
TABLE III

MAKeSPAN OF METhod II (10 JOB 10 MACHINE)

mean (variance)

\begin{tabular}{|l|c|c|c|}
\hline problem & $10 / 10$ & $3 / 10$ & $5 / 10$ \\
\hline abz5 & 1281 & $1308(5.3)$ & $1334(0.0)$ \\
\hline abz6 & 980 & $980(0.0)$ & $* 991(15.8)$ \\
\hline ft10 & 1007 & $1020(5.2)$ & $1020(5.2)$ \\
\hline la16 & 998 & ${ }^{*} 1148(33.2)$ & $* 1144(61.8)$ \\
\hline la17 & 823 & $834(15.6)$ & $836(0.0)$ \\
\hline la18 & 907 & ${ }^{*} 985(15.1)$ & $* 993(6.6)$ \\
\hline la19 & 896 & ${ }^{*} 1002(22.9)$ & ${ }^{*} 994(1.4)$ \\
\hline la20 & 949 & ${ }^{*} 1023(76.4)$ & $949(0.0)$ \\
\hline orb01 & 1196 & $1246(41.8)$ & $1196(0.0)$ \\
\hline orb02 & 921 & $978(24.0)$ & $1016(0.0)$ \\
\hline orb03 & 1147 & $* 1194(11.2)$ & $* 1194(11.2)$ \\
\hline orb04 & 1084 & $* 1252(32.2)$ & $* 1233(6.7)$ \\
\hline orb05 & 1015 & $* 1065(18.2)$ & $* 1097(10.6)$ \\
\hline
\end{tabular}

UJR, SPT and SLACK is again represented by

$$
\begin{aligned}
\min _{j} S_{k j} & =h_{1} p_{k j}+h_{2} \frac{s_{j}}{J_{m}}+\left(1-h_{1}-h_{2}\right) U_{j}, \\
0 & <h_{1}<1,0<h_{2}<1, h_{1}+h_{2}<1 .
\end{aligned}
$$

In this rule, we select a job with the smallest $S_{k j}$. This is a proposed method $I I$.

\section{B.2 Simulation of Method II-1}

We examined the best makespan when $h_{1}$ and $h_{2}$ change from 0 to 1 . Then we examined the best combination of $h_{1}$ and $h_{2}$ at $3 / 10$ and $5 / 10$ of the total processing time. After fixing $h_{1}$ and $h_{2}$, the rest of schedule is planned. The mean and the variance of the makespan are calculated for all conflicting schedules.

Table III shows the best makespan of the combined rule, the mean and the variance of the makespan with the best combination of $h_{1}$ and $h_{2}$ at the stage of $3 / 10$ and 5/10. The mark "*" denotes the mean of the makespan is longer than the conventional rule in Table II.

From Table III, many problems have the mark "*". It means that it is difficult to decide $h_{1}$ and $h_{2}$ in the early phase for small scale problems.

\section{B.3 Simulation of Method II-2}

Table IV presents simulation results for large scale problems with 100-job-100-machine. Other conditions are same as the case of the simulation of Method II-1. In this table, data5 to data16 are problems in which sequence of operations for each job are random. data21 to data30 are problems in which sequence of operations for each job are similar.

From Table IV, while a number of the mark "*" is fewer than Table III, some problems still have the mark, i. e., it is still difficult to decide $h_{1}$ and $h_{2}$ in early stage of the schedule. In addition, results of similar sequence problems are worse than results of random sequence problems. Table V shows conventional makespans of same problems. MWKR 
TABLE IV

TABLE VI

Makespan of Method II-1 (100-Job-100-MAChine)

mean (variance)

\begin{tabular}{|l|c|c|c|}
\hline problem & $10 / 10$ & $3 / 10$ & $5 / 10$ \\
\hline data5 & 12551 & $12643(52.6)$ & $12639(0.0)$ \\
\hline data6 & 12613 & $12767(0.0)$ & $12834(0.0)$ \\
\hline data7 & 12531 & $12740(36.8)$ & $12545(0.0)$ \\
\hline data8 & 12603 & $12783(0.0)$ & $12687(0.0)$ \\
\hline data9 & 12650 & $12966(23.0)$ & $12709(0.0)$ \\
\hline data11 & 10183 & $10254(41.5)$ & $10514(0.0)$ \\
\hline data16 & 12733 & $12904(0.0)$ & $12864(0.0)$ \\
\hline data21 & 24054 & $24192(144.7)$ & $24149(0.0)$ \\
\hline data22 & 24668 & $* 25961(149.0)$ & $24815(19.3)$ \\
\hline data23 & 23473 & $* 25030(0.0)$ & $* 24723(0.0)$ \\
\hline data25 & 21271 & $* 23973(0.0)$ & $21518(0.0)$ \\
\hline data28 & 20400 & $* 21882(0.0)$ & $21031(525.4)$ \\
\hline data29 & 19976 & $* 21119(0.0)$ & $20299(84.3)$ \\
\hline data30 & 16682 & $17245(0.0)$ & $17111(0.0)$ \\
\hline
\end{tabular}

TABLE V

CONVENTIONAL MAKESPAN

\begin{tabular}{|l|ll|}
\hline problem & \multicolumn{2}{|c|}{ conventional makespan } \\
\hline data5 & 12914 & $(\mathrm{MWKR})$ \\
\hline data6 & 12958 & $(\mathrm{LOPN})$ \\
\hline data7 & 12935 & $(\mathrm{MWKR})$ \\
\hline data8 & 12937 & $(\mathrm{LOPN})$ \\
\hline data9 & 12999 & $(\mathrm{MWKR})$ \\
\hline data11 & 10579 & $(\mathrm{MWKR})$ \\
\hline data16 & 13003 & $(\mathrm{MWKR})$ \\
\hline data21 & 25035 & $(\mathrm{SPT})$ \\
\hline data22 & 25098 & $(\mathrm{SPT})$ \\
\hline data23 & 23680 & $(\mathrm{SPT})$ \\
\hline data25 & 22084 & $(\mathrm{SPT})$ \\
\hline data28 & 21553 & $(\mathrm{SPT})$ \\
\hline data29 & 20618 & $(\mathrm{SPT})$ \\
\hline data30 & 18118 & $(\mathrm{MWKR})$ \\
\hline
\end{tabular}

tends to give good results for random sequence problems and SPT tends to give good results for similar sequence problems.

\section{B.4 Effect of Processing Sequence}

It is effective to apply SPT rule for early scheduled machines and to apply SLACK rule for late scheduled machines. Let $A_{k}$ be an index of earliness of processing order in operations in machine $k . A_{k}$ is defined as

$$
A_{k}=\frac{-\left(R_{k}-R_{A V E}\right) M_{k} t_{k}}{M_{A V E}},
$$

where $R_{k}$ denotes the average processing order in machine $k$ for all jobs. $R_{A V E}$ denotes the average of all $R_{k} . M_{k}$ denotes the number of remaining operations in machine $k . t_{k}$ denotes the average processing time of operations in machine $k$ for all jobs. $A_{k}>0$ means the average processing order in machine $k$ is early, and $A_{k}<0$ means the average
MaKeSPAN OF METHOD II-2 (100-JOB-100-MACHINE)

mean (variance)

\begin{tabular}{|l|c|c|c|}
\hline problem & $10 / 10$ & $3 / 10$ & $5 / 10$ \\
\hline data5 & 12557 & $12646(57.3)$ & $12646(57.3)$ \\
\hline data6 & 12656 & $12805(0.0)$ & $12841(0.0)$ \\
\hline data7 & 12601 & $12698(78.3)$ & $12834(62.9)$ \\
\hline data8 & 12597 & $12737(0.0)$ & $12736(89.7)$ \\
\hline data9 & 12651 & $12886(0.0)$ & $12686(46.4)$ \\
\hline data11 & 10174 & $10292(25.2)$ & $10234(11.0)$ \\
\hline data16 & 12804 & $12929(5.2)$ & $12952(0.0)$ \\
\hline data21 & 24255 & $24515(246.8)$ & $24439(227.3)$ \\
\hline data22 & 25023 & $* 26098(239.3)$ & $25017(0.0)$ \\
\hline data23 & 23515 & $* 23964(192.8)$ & $* 23902(46.1)$ \\
\hline data25 & 21460 & $22038(90.4)$ & $21962(84.6)$ \\
\hline data28 & 20484 & $20978(0.0)$ & $20978(0.0)$ \\
\hline data29 & 20275 & $20607(147.2)$ & $* 20645(166.7)$ \\
\hline data30 & 17022 & $17617(270.9)$ & $17744(31.9)$ \\
\hline
\end{tabular}

processing order in machine $k$ is late. The modified UJR rule considering the new factor $A_{k}$ is defined as

$$
U_{j}^{\prime}=\left|\left(l_{k}-p_{j}\right)-\left(\frac{l_{1}+l_{2}+\cdots+\left(l_{k}-p_{j}\right)+\cdots+l_{m}}{m}-A_{k}\right)\right| .
$$

Due to the factor $A_{k}$, if the average order in machine $k$ is early, SPT is preceded. If the average order in machine $k$ is middle, $A_{k}$ has a small value.

Table VI presents the result of the combined rule $U_{j}^{\prime}$. By comparing Table IV with Table VI, a number of the mark "*" is fewer than Table IV. Decrease of the mark "*" means an improvement in a combined rule. Therefore, this combined rule can give good results by determining the parameter $h_{1}$ and $h_{2}$ in early stage of the schedule.

\section{Proposed Method III}

From Table V, it is clear that MWKR is effective in random sequence problems, and SPT is effective in similar sequence problems. We propose the third combined rule that predetermine the parameter $h_{1}$ as follows.

C.1 Predetermination of Weight of Rules

In a proposed method III, $h_{1}$ is given by

$$
h_{1}=\frac{R_{k} / R_{A V E}}{2} .
$$

If $h_{1}$ is bigger than $1 / 2$, SPT becomes dominant. Otherwise, SLACK becomes dominant.

\section{C.2 Simulation of Method III}

Table VII and Table VIII present the result of the proposed method III for 10-job-10-machine and 100-job-100machine problems. In this table, a mark "\#" denotes that the makespan of the proposed method III is better than the best among the conventional four rules.

The proposed method III yields better makespans for 12 problems out of 27 problems, i. e., the method is more 


\section{TABLE VII}

MAKESPAN OF METHOD III (10 JOB 10 MACHINE)

\begin{tabular}{|l|c|cl|}
\hline problem & method III & \multicolumn{2}{|c|}{ conventional rule } \\
\hline abz5 & $1281 \#$ & 1352 & (SPT) \\
\hline abz6 & $981 \#$ & 987 & (MWKR) \\
\hline ft10 & $1074 \#$ & 1074 & (SPT) \\
\hline la16 & 1156 & 1054 & (MWKR) \\
\hline la17 & 913 & 846 & (MWKR) \\
\hline la18 & 981 & 970 & (MWKR) \\
\hline la19 & $940 \#$ & 940 & (SPT) \\
\hline la20 & 1000 & 964 & $($ MWKR) \\
\hline orb01 & 1330 & 1326 & $($ LOPN) \\
\hline orb02 & $996 \#$ & 1047 & $($ MWKR) \\
\hline orb03 & $1178 \#$ & 1179 & $($ SPT) \\
\hline orb04 & 1218 & 1132 & $($ LOPN) \\
\hline orb05 & $1044 \#$ & 1055 & $($ LOPN) \\
\hline
\end{tabular}

TABLE VIII

MAKESPAN OF METHOD III (100 JOB 100 MACHINE)

\begin{tabular}{|l|c|ll|}
\hline problem & method III & \multicolumn{2}{|c|}{ conventional rule } \\
\hline data5 & $12728 \#$ & 12914 & (MWKR) \\
\hline data6 & 12969 & 12958 & (LOPN) \\
\hline data7 & $12864 \#$ & 12935 & (MWKR) \\
\hline data8 & 13390 & 12937 & (LOPN) \\
\hline data9 & 13107 & 12999 & (MWKR) \\
\hline data11 & 10980 & 10579 & (MWKR) \\
\hline data16 & 13024 & 13003 & (MWKR) \\
\hline data21 & $24378 \#$ & 25035 & (SPT) \\
\hline data22 & 25853 & 25098 & (SPT) \\
\hline data23 & 24146 & 23680 & (SPT) \\
\hline data25 & 23536 & 22084 & (SPT) \\
\hline data28 & $21378 \#$ & 21553 & (SPT) \\
\hline data29 & 21409 & 20618 & (SPT) \\
\hline data30 & $17731 \#$ & 18118 & (MWKR) \\
\hline
\end{tabular}

general than the conventional rules. From the result, it is clear that the proposed method III is effective.

\section{Conclusion}

We have proposed three combined rules. The first rule is the rule that combines two simple dispatch rules which are often adopted in actual production systems. The proposed rule gives better result than that of any single dispatch rule.

The second rule is the rule that keeps balance of remaining load of all machines. In this rule, the weight parameter $h_{1}$ and $h_{2}$ is decided in early stage of the schedule. It gives good solutions with high probability. The method is effective in the case of large scale problems.

The third rule is the rule that predetermines the weight parameter $h_{1}$, which accompanies a sequence of operations. This rule often gives better results than any other dispatch rules. Since it is possible to decide the weight parameter $h_{1}$ before scheduling, method III is simple and

\section{REFERENCES}

[1] R. Haupt, "A Survey of Priority Rule-Based Scheduling", OR Spektrum, vol. 11, pp. 3-16 (1989)

[2] J. E. Beasley: "OR-Library: Distributing Test Problems by Electronic Mail", J. Opl. Res. Soc, Vol. 41, No. 11, pp. 1069-1072 (1990)

[3] S. A. Cook: "The Complexity of Theorem-Proving Procedures", Proceedings of the Third Annual ACM Symposium on Theory of Computing, pp. 151-158 (1971)

[4] R. M. Karp: "Reducibility among combinatorial problems", Complexity of Computation (R. E. Miller and J. W. Thacher, Eds.), Plenum Press, pp. 85-104 (1972)

[5] S. J. Morton and D. Pentico: "Heuristic Scheduling Systems", Wiley (1993)

[6] I. M. Ovacik and R. Uzsoy: "Decomposition Methods for Complex Factory Scheduling Problems", Kluwer Academic Publishers (1997) 